Pacific Journal of Mathematics

NILPOTENCY AND SPECTRAL OPERATORS 


\title{
NILPOTENCY AND SPECTRAL OPERATORS
}

\author{
JAMES E. SIMPSON
}

1. Introduction. The present paper is concerned with conditions under which the quasi-nilpotent part of a spectral operator is actually nilpotent of some order $k$. As might be expected, the case of a spectral operator on a Hilbert space has been settled longest. (See [4].) The case of a Banach space has been treated quite thoroughly by C. A. McCarthy [7] who showed that with a certain rate of growth condition on $Q$, the nilpotent part of the spectral operator $T=S+Q$, satisfies $Q^{m+2}=0$, where the $m$ is a positive integer involved in the rate of growth condition. He also discusses more special cases in which $Q^{m+1}=0$ and provides examples to show that these exponents are the lowest possible in each case. The question of extending these results to general locally convex spaces could not even be formulated until a theory of spectral operators in these spaces had been devised. The work of C. Ionescu Tulcea [5] having laid the foundations in this area, we may now attempt to solve the problem of generalizing McCarthy's results. It is shown below that his theorems, and indeed some part of the proofs, may be carried over to the locally convex case, with a suitable reformulation of some of the conditions and reworking of some of the supporting theory.

The basic assumptions are as follows. $E$ denotes a locally convex linear topological space over the field, $C$, of complex numbers. Moreover, $E$ is assumed to be separated, barrelled and quasi-complete. The strong dual of $E$ is denoted by $E^{\prime}$. The space of continuous linear mappings of $E$ into itself is $\mathscr{L}(E, E)$, which we shall always assume to be given the topology $\mathscr{T}_{b}$ of uniform convergence on the bounded subsets of $E$. We denote the adjoint of $T$ by ${ }^{t} T$, for each $T \in \mathscr{L}(E, E)$. The resolvent set of $T$, res $T$, is a certain subset of $\hat{C}$, the one-point compactification of $C$. Specifically, $\lambda \in \operatorname{res} T$ provided there is a neighborhood $V_{\lambda}$ of $\lambda$ in $\widehat{C}$ and a function $R_{T}$ with domain $V_{\lambda} \cap C$ and range in $\mathscr{L}(E, E)$ such that

(a) the set $\left\{R_{T}(z) x: z \in V_{\lambda} \cap C\right\}$ is a bounded subset of $E$ for each $x \in E$, and

(b) $R_{T}(z)(z I-T)=(z I-T) R_{T}(z)=I$ for all $z \in V_{\lambda} \cap C$. The complement of res $T$, in $\widehat{C}$, is the spectrum of $T$, denoted sp $T$. If $\infty \notin \mathrm{sp} T$, then sp $T$ is compact in $C$ and we say $T$ is regular. We

Received June 6, 1963. Most of the work appearing in this paper was done while the author was a National Science Foundation Graduate Fellow and appears in somewhat modified form in his dissertation, submitted to Yale University while he was a candidate for the doctoral degree. 
denote by $B^{\infty}(C)$ the algebra of bounded complex-valued Baire functions on $C$, with norm $\left|f \mathbf{W}_{u}=\sup _{z \in O}\right| f(z) \mid$, and by $S(C)$ the Baire sets, i.e., those subsets, $A$, of $C$ whose characteristic function, $\varphi_{A}$, is in $B^{\infty}(C)$. We denote by $M^{1}$ the set of bounded Radon measures on $C$, with norm $\mu=\sup \left\{\left|\int_{\sigma} f d \mu\right|: f \in B^{\infty}(C), \quad f \mathbf{U}_{u}=1\right\}$. By sup $\mu$ we mean the support of the measure $\mu$. Further information about locally convex spaces and Radon measures may be found in the well-known Bourbaki books [1] and [2]. Concerning the resolvent and spectrum of $T$, see L. Waelbroeck [10] and [11].

Turning now to spectral operators, we review some of the definitions and theorems to be found in the above mentioned work of Ionescu Tulcea [5]. See also F. Maeda [6] and H. Schaeffer [8]. The latter paper contains a monumental quantity of information about spectral measures and extensions and proofs of many of the observations listed in this paragraph. With $E$ as above, let $\mathscr{F}=\left\{\mu_{x, x^{\prime}}: x \in E, x^{\prime} \in E^{\prime}\right\}$ be a set of bounded Radon measures on $C$, indexed as indicated by $E \times E^{\prime}$. We say that $\mathscr{F}$ is a spectral family of measures if there is a continuous algebraic representation of $B^{\infty}(C)$ in $\mathscr{L}(E, E)$, denoted by $f \rightarrow U_{\mathscr{F}, f}$ (or $U_{f}$, if no confusion will ensue) such that $U_{l}=I$, and $\left\langle U_{f} x, x^{\prime}\right\rangle=\int_{0} f d \mu_{x, x^{\prime}}$ for all $x \in E, x^{\prime} \in E^{\prime}$, and $f \in B^{\infty}(C)$. The function $l$ is defined by $l(z)=1$ for all $z \in C$. By sup $\mathscr{F}$ we mean $\overline{\bigcup_{x \in E E} \sup \mu_{x, x^{\prime}}}$. We say that $T \in \mathscr{L}(E, E)$ commutes with a spectral family $\mathscr{F}$, provided $T U_{f}=U_{f} T$ for all $f \in B^{\infty}(C)$. Denote by $P_{\mathscr{F}}$ the $\mathscr{L}(E, E)$-valued function defined on $S(C)$ by $P_{\mathscr{F}}(\sigma)=U_{\varphi_{\sigma}}$. Then $P_{\mathscr{F}}$ has the following properties:

(i) $P_{\mathscr{F}}(\phi)=0$.

(ii) $P_{\mathscr{F}}(\sigma \cap \delta)=P_{\mathscr{F}}(\sigma) P_{\mathscr{F}}(\delta)$ for all $\sigma, \delta \in S(C)$.

(iii) The set function $m_{x}$, defined on $S(C)$ with values in $E$ by $m_{x}(\sigma)=P_{\mathscr{F}}(\sigma) x$, is countably additive for each $x \in E$.

(iv) $P_{\mathscr{F}}(C)=I$.

We shall call $P_{\mathscr{F}}$ the spectral measure associated with $\mathscr{F}$. It is quite common to write $\int_{\sigma} f d P_{\mathscr{F}}$ for $U_{f}$. For each $\sigma \in S(C)$, let $E_{\sigma}=P_{\mathscr{F}}(\sigma) E$. If $T \in \mathscr{L}(E, E)$ commutes with $\mathscr{F}$, then $T_{\sigma}: E_{\sigma} \rightarrow E_{\sigma}$ may be defined by $T_{\sigma} x=T x$ for all $x \in E_{\sigma}$. An element $T$ of $\mathscr{L}(E, E)$ is said to be a spectral operator if there is a spectral family $\mathscr{F}\left(=\mathscr{F}_{T}\right.$, if necessary) on $C$ such that $T$ commutes with $\mathscr{F}$ and sp $T_{\sigma} \subset \sigma$ for every compact subset $\sigma$ of $C$. An element $Q$ of $\mathscr{L}(E, E)$ is said to be quasi-nilpotent if $\operatorname{limit}_{n}\left|\left\langle Q^{n} x, x^{\prime}\right\rangle\right|^{1 / n}=0$ for all $x \in E, x^{\prime} \in E^{\prime}$. An element $S$ of $\mathscr{L}(E, E)$ is said to be scalar relative to a spectral family $\mathscr{F}$ if the function $f: f(z)=z$ is $\mu_{x, x^{\prime}}$-measurable for all $\mu_{x, x^{\prime}} \in \mathscr{F}$ and $\left\langle S x, x^{\prime}\right\rangle=\int_{O} f d \mu_{x, x^{\prime}}$ for all $x \in E, x^{\prime} \in E^{\prime}$. Finally we mention con- 
dition $P \mathscr{C}$ ), which is described in [5] and [6]. The central decomposition theorem of spectral theory then is that if $T$ is a spectral operator whose associated spectral family $\mathscr{F}_{T}$ satisfies condition $P \mathscr{C}$ ), there is a unique decomposition $T=S+Q$ where $S$ is scalar relative to $\mathscr{F}_{T}$ and $Q$ is quasi-nilpotent.

One last tool will be needed below, namely an "operational calculus" for regular operators. Suppose $T$ is regular. Let $\mathscr{G}(T)$ be the class of all complex-valued functions $f$, analytic on an open set $D(f)$ which contains sp $T$. Let $D$ be any Cauchy domain satisfying sp $T \subset D \subset \bar{D} \subset D(f)$. Then for each $f \in \mathscr{G}(T)$, define $f(T)=$ $1 / 2 \pi i \int_{\Gamma} f(z) R_{T}(z) d z$, where $\Gamma$ is the boundary of $D$. We then have the following theorem.

THEOREM 1. For any $f \in \mathscr{G}(T), f(T)$ is a well-defined element of $\mathscr{L}(E, E)$ independent of the choice of $D$ (provided it satisfies the above conditions). Moreover, if $f$ and $g$ are both in $\mathscr{G}(T)$, then $(f+g)(T)=f(T)+g(T)$ and $(f g)(T)=f(T) g(T)$. If $\Gamma$ is a circle of sufficiently large radius to contain sp $T$ in its interior, then $T=1 / 2 \pi i \int_{\Gamma} z R_{T}(z) d z$ and $I=1 / 2 \pi i \int_{\Gamma} R_{T}(z) d z$. Finally,

$$
\left\langle\int_{\Gamma} f(z) R_{T}(z) d z x, x^{\prime}\right\rangle=\int_{\Gamma}\left\langle f(z) R_{T}(z) x, x^{\prime}\right\rangle d z
$$

for every $x \in E, x^{\prime} \in E^{\prime}$, and $f \in \mathscr{G}(T), \Gamma \subset D(f)$.

The definition of Cauchy domain is to be found in Taylor's paper [9], which also contains a proof of a theorem very similar to the above which may easily be adjusted to fit the present situation. The theorem might also be considered a special case of some of the work of H. Cartan [3]. With this background we are prepared to discuss conditions under which $Q^{k}=0$ for some positive integer $k$.

2. The general case. Let $E$ be a separated, locally convex space which is barrelled and quasi-complete. Let $T$ be a spectral operator on $E$ whose spectral family $\mathscr{F}$ satisfies condition $P \mathscr{C}$ ) so that we may write $T=S+Q$. We now state our rate of growth condition:

Definition. With $E$ and $T$ as described, we say that $R_{T}$ satisfies an $m$ th-order rate of growth condition ( $m$ being a positive integer) if the set $\left\{d(z, \sigma)^{m} R_{T_{\sigma}}(z) P_{\mathscr{F}}(\sigma): z \notin \sigma, \sigma\right.$ compact $\}$ is bounded in $(\mathscr{L}(E, E)$, $\left.\mathscr{T}_{b}\right)$. Here $d(z, \sigma)$ is the distance from $z$ to $\sigma$.

For the rest of this section we assume that $R_{T}$ satisfies an $m$ thorder rate of growth of condition. 
LEMma 1. Let $n$ be a fixed positive integer, $\sigma$ a compact subset of $C, V$ a neighborhood of zero in $\mathscr{L}(E, E)$. Then there is a finite partition of $\sigma$ by Borel sets $\left\{\sigma_{j}: j=1,2, \cdots, k\right\}$ with the property that if $\left\{\lambda_{j}: j=1,2, \cdots, k\right\}$ is any choice of $k$ complex numbers with. each $\lambda_{j} \in \sigma_{j}$, then

$$
Q^{n} P_{\mathscr{F}}(\sigma)-\sum_{j}\left(T-\lambda_{j}\right)^{n} P_{\mathscr{F}}\left(\sigma_{j}\right) \in V .
$$

Proof. Denoting by $f_{i}$ the function $f_{i}(z)=z^{i} \varphi_{\sigma}(z)$, for $i=1,2, \cdots$, $n$, we observe that for every $\varepsilon>0$ there is a partition $\left\{\sigma_{j}: j=\right.$ $1,2, \cdots, k\}$ of $\sigma$ such that $\sum_{j} \lambda_{j}^{i} \varphi_{\sigma_{j}}-f_{i} \mathbf{\square}_{u}<\varepsilon$ for all $i=1,2, \cdots, n$, where the $\lambda_{j}$ are arbitrary in $\sigma_{j}$. Next, since the mapping $f \rightarrow U_{f}$ is continuous, it follows that $S^{i} P_{\mathscr{F}}(\sigma)=U_{f_{i}}$ may be approximated in the topology of $\mathscr{L}(E, E)$ by operators of the form $\sum_{j} \lambda_{j}^{i} P_{\mathscr{F}}\left(\sigma_{j}\right)$, uniformly for $i=1,2, \cdots, n$, and for $\lambda_{j} \in \sigma_{j}$. The theorem may now be proved by considering

$$
Q^{n} P_{\mathscr{F}}(\sigma)=(T-S)^{n} P_{\mathscr{F}}(\sigma)=\sum_{i=0}^{n}(-1)^{i}\left(\begin{array}{c}
n \\
i
\end{array}\right) T^{n-i} S^{i} P_{\mathscr{F}}(\sigma) .
$$

Given $V$, choose $U$, an equilibrated neighborhood of zero in $\mathscr{L}(E, E)$, such that $\sum_{i=0}^{n}\left(\begin{array}{l}n \\ i\end{array}\right) U \subset V$. For each $i=0,1, \cdots, n$, choose a neighborhood $W_{i}$ of zero in $\mathscr{L}(E, E)$ such that $A \in W_{i}$ implies $T^{n-i} A \in U$. Finally, choose $\left\{\sigma_{j}\right\}$ so that $\sum_{j} \lambda_{j}^{i} P_{\mathscr{F}}\left(\sigma_{j}\right)-S^{i} P_{\mathscr{F}}(\sigma) \in \cap i W_{i}$. Then,

$$
\sum_{i}(-1)^{i}\left(\begin{array}{c}
n \\
i
\end{array}\right) \sum_{j} T^{n-i} \lambda_{j}^{i} P_{\mathscr{F}}\left(\sigma_{j}\right)-\sum_{i}(-1)^{i}\left(\begin{array}{c}
n \\
i
\end{array}\right) T^{n-i} S^{i} P_{\mathscr{F}}(\sigma) \in V .
$$

But the first term in this last expression is just $\sum_{j}\left(T-\lambda_{j}\right)^{n} P_{\mathscr{F}}\left(\sigma_{j}\right)$. so that (1) is proved.

LEMMA 2. For every bounded subset $B$ of $E$, every equicontinuous subset $B^{\prime}$ of $E^{\prime}$, and every positive integer $n$, there is a positive real number $M=M\left(B, B^{\prime}, n\right)$ such that

$$
\left|\left\langle Q^{n} P_{\mathscr{F}}(\sigma) x, x^{\prime}\right\rangle\right| \leqq M \varepsilon^{n-m+1}
$$

for all $x \in B, x^{\prime} \in B^{\prime}$, provided $0<\varepsilon \leqq 1$ and $\sigma$ is a Borel set of diameter $\leqq \varepsilon$.

Proof. If $\sigma$ is empty there is no problem. Next consider the case where $\sigma$ is a nonvoid compact subset of $C$, and fix $\eta>0$. By Lemma 1 there is a partition $\left\{\sigma_{j}\right\}$ of $\sigma$ such that

$$
\left|\left\langle Q^{n} P_{\mathscr{F}}(\sigma) x, x^{\prime}\right\rangle-\left\langle\sum_{j}\left(T-\lambda_{j}\right)^{n} P_{\mathscr{F}}\left(\sigma_{j}\right) x, x^{\prime}\right\rangle\right|<\eta
$$

for all $x \in B, x^{\prime} \in B^{\prime}$. Using any point in $\sigma$ as center, construct a 
circle, $\Gamma$, of radius $2 \varepsilon$. Then $|z-\lambda| \geqq \varepsilon$ for all $z \in \Gamma, \lambda \in \sigma$. Then, for all $x \in B, x^{\prime} \in B^{\prime}$,

$$
\begin{aligned}
\left|\left\langle\sum_{j}\left(T-\lambda_{j}\right)^{n} P_{\mathscr{F}}\left(\sigma_{j}\right) x, x^{\prime}\right\rangle\right| & \\
= & \left|\left\langle\sum_{j} \frac{1}{2 \pi i} \int_{\Gamma}\left(z-\lambda_{j}\right)^{n} R_{T_{\sigma}}(z) d z P_{\mathscr{F}}\left(\sigma_{j}\right) x, x^{\prime}\right\rangle\right| \\
& =\frac{1}{2 \pi}\left|\int_{\Gamma}\left\langle\sum_{j}\left(z-\lambda_{j}\right)^{n} R_{T_{\sigma}}(z) P_{\mathscr{F}}\left(\sigma_{j}\right) x, x^{\prime}\right\rangle d z\right| \\
& \leqq \frac{1}{2 \pi} L(\Gamma) \sup _{z \in \Gamma}\left|\left\langle\sum_{j}\left(z-\lambda_{j}\right)^{n} P_{\mathscr{F}}\left(\sigma_{j}\right) R_{T_{\sigma}}(z) P_{\mathscr{F}}(\sigma) x, x^{\prime}\right\rangle\right| \\
& =2 \varepsilon \sup _{z \in \Gamma}\left|\left\langle\int_{\sigma} \sum_{j}\left(z-\lambda_{j}\right)^{n} \varphi_{\sigma_{j}}(\lambda) d P_{\mathscr{F}}(\lambda) R_{T_{\sigma}}(z) P_{\mathscr{F}}(\sigma) x, x^{\prime}\right\rangle\right| .
\end{aligned}
$$

Let $g_{z}(\lambda)=\sum_{j}\left(z-\lambda_{j}\right)^{n} \varphi \sigma_{j}(\lambda)$ be the integrand function in the spectral integral in this last expression. Then the above computation implies

$$
\left|\left\langle\sum_{j}\left(T-\lambda_{j}\right)^{n} P_{\mathscr{F}}\left(\sigma_{j}\right) x, x^{\prime}\right\rangle\right| \leqq 2 \varepsilon \sup _{z \in \Gamma}\left|\left\langle U_{g_{z}} R_{T_{\sigma}}(z) P_{\mathscr{F}}(\sigma) x, x^{\prime}\right\rangle\right| .
$$

Since $\sup _{z \in \Gamma} \square g_{z} \mathbf{U}_{u} \leqq(3 \varepsilon)^{n}$ and the set $\left\{U_{f}: \square f \mathbf{W}_{u} \leqq 1\right\}$ is equicontinuous, corresponding to $B^{\prime}$ we may find a neighborhood $W$ of zero in $E$ such that

$$
\left|\left\langle U_{g_{z}} y, x^{\prime}\right\rangle\right| \leqq(3 \varepsilon)^{n}
$$

for all $y \in W$, and $x^{\prime} \in B^{\prime}$. It is apparent that $W$ is independent of $\varepsilon$, of $\sigma$, of the choice of $\left\{\sigma_{j}\right\}$, and of $z \in \Gamma$. But $B$ and $W$ determine a neighborhood of zero in $\mathscr{L}(E, E)$. Consequently, from the rate of growth condition, there is $\alpha>0$ such that

$$
d(z, \sigma)^{m} \alpha^{-1} R_{T_{\sigma}}(z) P_{\mathscr{F}}(\sigma) x \in W
$$

for all $x \in B, \alpha$ being dependent only on $B$ and $B^{\prime}$ (by way of $W$ ).

Substituting (6) in (5) gives, for all $z \in \Gamma$,

$$
\left|\left\langle U_{g_{z}} R_{T_{\sigma}}(z) P_{\mathscr{F}}(\sigma) x, x^{\prime}\right\rangle\right| \leqq \alpha(3 \varepsilon)^{n} d(z, \sigma)^{-m} \leqq \alpha 3^{m} \varepsilon^{n-m} .
$$

Letting $M=2 \cdot 3^{n} \alpha$ and substituting (7) in (4) and (3) we have

$$
\begin{aligned}
\left|\left\langle Q^{n} P_{\mathscr{F}}(\sigma) x, x^{\prime}\right\rangle\right| & \leqq\left|\left\langle\sum_{j}\left(T-\lambda_{j}\right)^{n} P_{\mathscr{F}}\left(\sigma_{j}\right) x, x^{\prime}\right\rangle\right|+\eta \\
& \leqq M \varepsilon^{n-m+1}+\eta
\end{aligned}
$$

for all $x \in B, x^{\prime} \in B^{\prime}$, with $M$ dependent only on $B, B^{\prime}$, and $n$. Since $\eta$ is arbitrary, the theorem is proved under the additional assumption that $\sigma$ be compact. However, the general case may be readily deduced from this one.

THEOREM 2. Let $\sigma$ be a Borel set in $C$ whose Hausdorff p-mea- 
sure is zero for some $p$. Then, for all $k \geqq p+m-1, Q^{k} P_{\mathscr{F}}(\sigma)=0$.

Proof. The hypothesis asserts that for every $\varepsilon>0$ there is a partition $\left\{\sigma_{j}\right\}$ of $\sigma$ by finitely or countably many Borel sets of diameters $\varepsilon_{j}$ respectivly such that $\varepsilon_{j} \leqq 1$ for all $j$ and $\sum_{j} \varepsilon_{j}^{p}<\varepsilon$. Let $k=p+m-1$. Then

$$
\left|\left\langle Q^{k} P_{\mathscr{F}}(\sigma) x, x^{\prime}\right\rangle\right| \leqq \sum_{j}\left|\left\langle Q^{k} P_{\mathscr{F}}\left(\sigma_{j}\right) x, x^{\prime}\right\rangle\right| \leqq \sum_{j} M \varepsilon_{i}^{k+1-m} \leqq M \varepsilon,
$$

where $M$ depends on $x, x^{\prime}$, and $k$. Since $\varepsilon, x$, and $x^{\prime}$ are arbitrary, we are done.

THEOREM 3. $Q^{m+2}=0$.

Proof. Let $p=3, \sigma=\sup \mathscr{F}$, and $k=3+m-1=m+2$ in the previous theorem.

3. Variations on the theme. In the case where $E$ is a Banach space, McCarthy has pointed out a number of variations on Theorem 3. The simplest of these, equally valid in our locally convex setting, flow directly from Theorem 2 when the $p$-measure of sp $T$ or of sup $\mathscr{F}$ is 0 for $p=1$ or 2 . An entirely different type of variation (also considered in [7]) may be discovered by observing that certain well-known Banach spaces may be embedded in $E$ or $E^{\prime}$. As in part 2 we assume that $T=S+Q$ is a spectral operator whose resolvent satisfies an $m$ th-order rate of growth condition.

Theorem 4. For every $x \in E$, and $x^{\prime} \in E^{\prime}$, the measure $\mu_{Q^{m+1} x, x^{\prime}}$ has base Lebesgue planar measure, $\lambda$. In fact, for every bounded set $B$ in $E$, and equicontinuous set $B^{\prime}$ in $E^{\prime}$, there is $N=N\left(B, B^{\prime}\right)$ such that for all Borel sets $\sigma$, all $x \in B$, and all $x^{\prime} \in B^{\prime}$, we have

$$
\mu_{Q^{m+1} x, x^{\prime}}(\sigma) \leqq N \lambda(\sigma) \text {. }
$$

Proof. Actually we prove this for $\mu_{2}$, Hausdorff 2-measure in the plane, but this is equivalent to proving it for $\lambda$. Let $\sigma$ be a Borel set in the plane of finite $\mu_{2}$ measure, and $\varepsilon>0$. Partition $\sigma$ by Borel sets $\left\{\sigma_{i}\right\}$ such that the diameter of $\sigma_{i}$ is less than $\varepsilon_{i}$, with $0<\varepsilon_{i} \leqq 1$, and $\sum_{i} \varepsilon_{i}^{2}<\mu_{2}(\sigma)+\varepsilon$. Letting $n=m+1$ in Lemma 2, we find that for each $B, B^{\prime}$ as described in the Theorem, there is $M>0$ such that $\left|\left\langle Q^{m+1} P_{\mathscr{F}}\left(\sigma_{i}\right) x, x^{\prime}\right\rangle\right| \leqq M \varepsilon_{i}^{2}$ for all $x \in B, x^{\prime} \in B^{\prime}$, and $i$. Consequently, $\left|\left\langle Q^{m+1} P_{\mathscr{F}}(\sigma) x, x^{\prime}\right\rangle\right| \leqq \sum_{i} M \varepsilon_{i}^{2} \leqq M\left(\mu_{2}(\sigma)+\varepsilon\right)$ for all $x \in B$, and $x^{\prime} \in B^{\prime}$. Since $\varepsilon$ is arbitrary, we are done.

We now denote by $g_{x, x^{\prime}}$ the Radon-Nikodym derivative of $\mu_{Q^{m+1} x, x^{\prime}}$ with respect to $\lambda$. If $f$ and $g$ are two elements of $B^{\infty}(C)$ which agree 
$\lambda$-almost everywhere, then for all $x \in E, x^{\prime} \in E^{\prime}$, we have

$$
\left|\left\langle U_{f-g} Q^{m+1} x, x^{\prime}\right\rangle\right|=\left|\int_{O}(f-g) g_{x, x^{\prime}} d \lambda\right|=0 .
$$

Hence $U_{f} Q^{m+1}=U_{g} Q^{m+1}$. Moreover, if $\left\{f_{n}\right\}$ is a sequence of simple functions which converges to $f$ in $L_{1}(C)$, then $U_{f_{n}} Q^{m+1}$ is a sequence which converges in $\mathscr{L}(E, E)$ to an operator which we denote by $U_{f} Q^{m+1}$. In this case $\left\langle U_{f} Q^{m+1} x, x^{\prime}\right\rangle=\int_{\sigma} f d \mu_{Q^{m+1} x, x^{\prime}}$.

We have already seen that $Q^{m+2}=0$. If we now assume $Q^{m+1} \neq 0$, by letting $\sigma=\sup \mathscr{F}$, we see that for some $x, x^{\prime}, \mu_{Q^{m+1} x, x^{\prime}}(\sigma) \neq 0$. Consequently there is a set $\tau$, compact if necessary, with nonvoid interior and a number $a>0$, such that $\lambda(\tau) \neq 0$ and $\left|g_{x, x^{\prime}}(z)\right|>a$ for all $z \in \tau$.

These two constructions are the basis for the embedding procedures mentioned above. The basic idea is to assume $E$ has some property which is inherited by all of its closed linear subspaces. Then, if $Q^{m+1} \neq 0$, we may embed a suitable space (perhaps an $L_{p}$-space) in $E$ or $E^{\prime}$ which does not have the property, thus obtaining a contradiction. In a written communication, McCarthy has suggested that $C(\tau)$ would be better than the $L_{\infty}(\tau)$ used in his paper for the case where $E$ is assumed weakly complete. As an indication of some of the details involved in such a construction, we prove here the following:

THEOREM 5. If $E$ is semi-reflexive, then $Q^{m+1}=0$.

Proof. As indicated, we assume $Q^{m+1} \neq 0$ and construct $\tau$ and $a$ corresponding to some $x_{0}, x_{0}^{\prime}$. Define $\Phi: L_{1}(\tau) \rightarrow E$ by the formula

$$
\Phi(f)=U_{\varphi_{\varphi_{\tau}}} Q^{m+1} x_{0} .
$$

Fix $\varepsilon>0$ and let $B^{\prime}$ be an arbitrary equicontinuous subset of $E^{\prime}$. Then for any $x^{\prime} \in B^{\prime}$,

$$
\begin{aligned}
\left|\left\langle\Phi(f), x^{\prime}\right\rangle\right| & =\left|\left\langle U_{f_{\tau}} Q^{m+1} x_{0}, x^{\prime}\right\rangle\right| \\
& =\left|\int_{\tau} f d \mu_{Q^{m+1} x_{0}, x^{\prime}}\right| \leqq N \boldsymbol{W} \mathbf{\bigsqcup}_{L_{1}(\tau)},
\end{aligned}
$$

where $N$ is chosen according to Theorem 4 with $B=\left\{x_{0}\right\}$. Thus $\left|\left\langle\Phi(f), x^{\prime}\right\rangle\right|<\varepsilon$ for all $x^{\prime} \in B^{\prime}$ whenever $f \mathbf{\bigsqcup}_{L_{1}}<\varepsilon / N$. Since $B^{\prime}$ is arbitrary, $\Phi$ is continuous. To see that $\Phi$ is one-to-one, first define $f^{*}$ for each $f$ in $L_{1}^{0}(\tau)$ by

$$
f^{*}(z)=\left\{\begin{array}{lll}
\overline{f(z)} /|f(z)| & \text { when } & f(z) \neq 0, \\
0 & \text { when } f(z)=0 .
\end{array}\right.
$$


Then $f^{*}$ is in $B^{\infty}(C)$ so that we may define $y^{\prime}=\left(\int_{\tau} f^{*} / g_{x_{0}, x_{0}^{\prime}} d P_{\mathscr{F}}\right) x_{0}^{\prime}$. Then $\left\langle\Phi(f), y^{\prime}\right\rangle=\int_{\tau} f f^{*} d \lambda=\int_{\tau}|f| d \lambda=\mathbb{\|} f \mathbf{|}_{L_{1}(\tau)}$. Thus the kernel of $\Phi$ is zero. To show that $\Phi^{-1}$ is continuous on the range of $\Phi$ it is sufficient to find, for each $\varepsilon>0$, an equicontinuous set $B^{\prime}$ in $E^{\prime}$ such that $\llbracket f \mathbf{\square}_{L_{1}}<\varepsilon$ whenever $\left|\left\langle\Phi(f), x^{\prime}\right\rangle\right| \leqq \varepsilon$ for all $x^{\prime} \in B^{\prime}$. The set

$$
B=\left\{\int_{\tau} f^{*} / g_{x_{0}, x_{0}^{0}} d P_{\mathscr{F}^{*}}: f \in L_{1}^{0}(\tau)\right\}
$$

is an equicontinuous subset of $\mathscr{L}(E, E)$. Consequently the set $B^{\prime}=$ $\left\{{ }^{t} A x_{0}^{\prime}: A \in B\right\}$ is equicontinuous in $E^{\prime}$. Finally,

$$
\begin{aligned}
\boldsymbol{\|} f \mathbf{U}_{L_{1}(\tau)} & =\left|\int_{\tau}\right| f\left|/ g_{x_{0}, x_{0}} d \mu_{Q^{m+1} x_{0}, x_{0}^{\prime}}\right| \\
& =\left|\left\langle U_{f_{\varphi_{\tau}}} Q^{m+1} x_{0},\left(\int_{\tau} f^{*} / g_{x_{0}, x_{0}^{\prime}} d P_{\mathscr{F}}\right) x_{0}^{\prime}\right\rangle\right| \\
& \leqq \sup _{A \in B}\left|\left\langle\Phi(f),{ }^{t} A x_{0}\right\rangle\right|=\sup _{x^{\prime} \in B^{\prime}}\left|\left\langle\Phi(f), x^{\prime}\right\rangle\right| .
\end{aligned}
$$

Consequently, $\Phi\left(L_{1}(\tau)\right)$ is a closed linear subspace of $E$ which is isomorphic in both algebraic and topological sense to $L_{1}(\tau)$. This contradicts the assumption that $E$ is semi-reflexive, hence the Theorem is proved.

\section{BIBLIOGRAPHY}

1. N. Bourbaki, Espaces vectoriels topologiques, Elements de Mathematique, Paris, (1953-55).

2. - Intégration. Elements de Mathematique, Paris (1952-60).

3. H. Cartan, Theorie spectrale des C-algébres commutatives, Seminaire Bourbaki, Exposé 125 (1956).

4. N. Dunford, Spectral operators, Pacific J. Math., 4 (1954), 321-354.

5. C. Ionescu Tulcea, Spectral operators on locally convex spaces, Bull. Amer. Math. Soc., 67 (1961), 125-128.

6. F. Maeda, $A$ characterization of spectral operators on locally convex spaces, Math. Annalen, 143 (1961), 59-74.

7. C. A. McCarthy, The nilpotent part of a spectral operator, Pacific J. Math., 9 (1959), 1223-1231.

8. H. Schaeffer, Spectral measures in locally convex algebras, Acta Math., 107 (1962), 125-173.

9. A. E. Taylor, Spectral theory of closed distributive operators, Acta Math., 84 (1950), 189-224.

10. L. Waelbroeck, Le calcul symbolique dans les algébres commutatives, J. Math. Pures Appl., 9e Série, 33 (1954), 147-186.

11. - Locally convex algebras: Spectral Theory, Seminar on complex analysis, Institute for Advanced Study, 1958, unpub.

MARQUETTE UNIVERSITY 


\title{
PACIFIC JOURNAL OF MATHEMATICS
}

\author{
EDITORS
}

\author{
Robert Osserman \\ Stanford University \\ Stanford, California \\ M. G. Arsove \\ University of Washington \\ Seattle 5, Washington
}

\author{
J. Dugundji \\ University of Southern California \\ Los Angeles 7, California \\ Lowell J. Paige \\ University of California \\ Los Angeles 24, California
}

\section{ASSOCIATE EDITORS}
E. F. BECKENBACH
B. H. NEUMANN
F. WOLF
K. YOSHIDA

\section{SUPPORTING INSTITUTIONS}

\author{
UNIVERSITY OF BRITISH COLUMBIA \\ CALIFORNIA INSTITUTE OF TECHNOLOGY \\ UNIVERSITY OF CALIFORNIA \\ MONTANA STATE UNIVERSITY \\ UNIVERSITY OF NEVADA \\ NEW MEXICO STATE UNIVERSITY \\ OREGON STATE UNIVERSITY \\ UNIVERSITY OF OREGON \\ OSAKA UNIVERSITY \\ UNIVERSITY OF SOUTHERN CALIFORNIA
}

\author{
STANFORD UNIVERSITY \\ UNIVERSITY OF TOKYO \\ UNIVERSITY OF UTAH \\ WASHINGTON STATE UNIVERSITY \\ UNIVERSITY OF WASHINGTON \\ AMERICAN MATHEMATICAL SOCIETY \\ CALIFORNIA RESEARCH CORPORATION \\ SPACE TECHNOLOGY LABORATORIES \\ NAVAL ORDNANCE TEST STATION
}

Mathematical papers intended for publication in the Pacific Journal of Mathematics should by typewritten (double spaced), and on submission, must be accompanied by a separate author's résumé. Manuscripts may be sent to any one of the four editors. All other communications to the editors should be addressed to the managing editor, L. J. Paige at the University of California, Los Angeles 24, California.

50 reprints per author of each article are furnished free of charge; additional copies may be obtained at cost in multiples of 50 .

The Pacific Journal of Mathematics is published quarterly, in March, June, September, and December. Effective with Volume 13 the price per volume (4 numbers) is $\$ 18.00$; single issues, $\$ 5.00$. Special price for current issues to individual faculty members of supporting institutions and to individual members of the American Mathematical Society: $\$ 8.00$ per volume; single issues $\$ 2.50$. Back numbers are available.

Subscriptions, orders for back numbers, and changes of address should be sent to Pacific Journal of Mathematics, 103 Highland Boulevard, Berkeley 8, California.

Printed at Kokusai Bunken Insatsusha (International Academic Printing Co., Ltd.), No. 6, 2-chome, Fujimi-cho, Chiyoda-ku, Tokyo, Japan.

PUBLISHED BY PACIFIC JOURNAL OF MATHEMATICS, A NON-PROFIT CORPORATION

The Supporting Institutions listed above contribute to the cost of publication of this Journal, but they are not owners or publishers and have no responsibility for its content or policies. 


\section{Pacific Journal of Mathematics}

\section{Vol. 14, No. $2 \quad$ June, 1964}

Tom M. (Mike) Apostol and Herbert S. Zuckerman, On the functional equation $F(m n) F((m, n))=F(m) F(n) f((m, n)) \ldots \ldots \ldots \ldots \ldots \ldots \ldots \ldots \ldots \ldots \ldots$

Reinhold Baer, Irreducible groups of automorphisms of abelian groups . . . . . . . 385

Herbert Stanley Bear, Jr., An abstract potential theory with continuous kernel . . . . 407

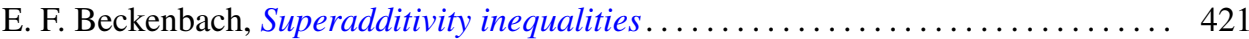

R. H. Bing, The simple connectivity of the sum of two disks . . . . . . . . . . . 439

Herbert Busemann, Length-preserving maps ...................... 457

Heron S. Collins, Characterizations of convolution semigroups of measures . . . . . . 479

Paul F. Conrad, The relationship between the radical of a lattice-ordered group and complete distributivity............................ 493

P. H. Doyle, III, A sufficient condition that an arc in $S^{n}$ be cellular . . . . . . . . . 501

Carl Clifton Faith and Yuzo Utumi, Intrinsic extensions of rings . . . . . . . . . . 505

Watson Bryan Fulks, An approximate Gauss mean value theorem . . . . . . . . . . 513

Arshag Berge Hajian, Strongly recurrent transformations . . . . . . . . . . . . . 517

Morisuke Hasumi and T. P. Srinivasan, Doubly invariant subspaces. II . . . . . . . 525

Lowell A. Hinrichs, Ivan Niven and Charles L. Vanden Eynden, Fields defined by

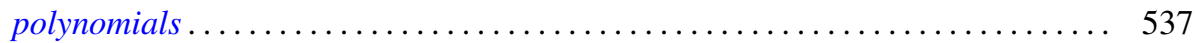

Walter Ball Laffer, I and Henry B. Mann, Decomposition of sets of group

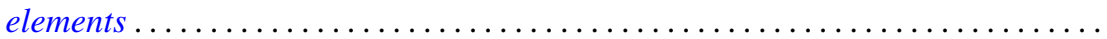

John Albert Lindberg, Jr., Algebraic extensions of commutative Banach

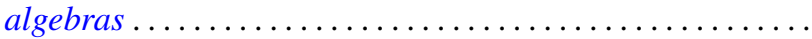

W. Ljunggren, On the Diophantine equation $C x^{2}+D=y^{n} \ldots$

M. Donald MacLaren, Atomic orthocomplemented lattices ....

Moshe Marcus, Transformations of domains in the plane and applications in the

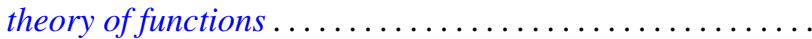

Philip Miles, $B^{*}$ algebra unit ball extremal points . .................. 627

W. F. Newns, On the difference and sum of a basic set of polynomials . . . . . . . 639

Barbara Osofsky, Rings all of whose finitely generated modules are injective ...... 645

Calvin R. Putnam, Toeplitz, matrices and invertibility of Hankel matrices . . . . . . . 651

Shoichiro Sakai, Weakly compact operators on operator algebras . . . . . . . . . 659

James E. Simpson, Nilpotency and spectral operators . . . . . . . . . . . . . 665

Walter Laws Smith, On the elementary renewal theorem for non-identically

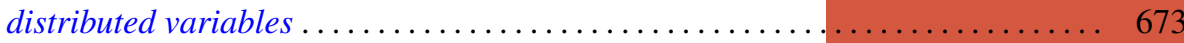

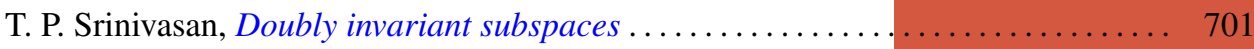

J. Roger Teller, On the extensions of lattice-ordered groups . . . . . . . . . . . . 709

Robert Charles Thompson, Unimodular group matrices with rational integers as

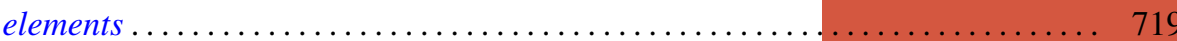

J. L. Walsh and Ambikeshwar Sharma, Least squares and interpolation in roots of unity

Charles Edward Watts, A Jordan-Hölder theorem .................... 731

Kung-Wei Yang, On some finite groups and their cohomology .............. 735

Adil Mohamed Yaqub, On the ring-logic character of certain rings ............ 741

Paul Ruel Young, A note on pseudo-creative sets and cylinders 